\title{
Persistence of symptoms after gall bladder clearance with cholecystolithotripsy
}

\author{
S H Lee, H J Burhenne
}

\begin{abstract}
Forty six patients rendered stone and fragment free with successful cholecystolithotripsy without the use of oral chemolitholysis were followed by serial ultrasound for a period of two to 25 months (mean 11 months, median 10 months). Seven patients (15\%), six of whom remain asymptomatic, developed recurrent calculi. Of the 39 gall stone free patients, 26 were asymptomatic. Six patients complained of persistent abdominal pain similar to that before treatment. One of these had a bile duct stone. Seven patients complained of a variety of non-biliary symptoms. The patient with recurrent gall stones and recurrent symptoms is eligible for further lithotripsy treatment. The overall $30 \%$ incidence of persistent abdominal symptoms is similar to that reported after elective cholecystectomy.
\end{abstract}

Cholecystolithotripsy has been used to treat patients with symptomatic gall stones at the University of British Columbia and Vancouver General Hospital since 1988. One of the major criticisms raised against this form of treatment is that the diseased gall bladder is left in situ, which may predispose to both stone recurrence and gall bladder carcinoma in the long term. Despite these obvious criticisms, the advantages of cholecystolithotripsy are that the patient does not require admission to hospital, a major operation is not performed, and a prolonged period of recuperation is not necessary. In addition, no mortality attributable to this form of noninvasive treatment for gall bladder stones has been reported to date.

Many lithotripsy centres treat only between $15-25 \%$ of the patients referred, because of their strict entry criteria. ${ }^{1}$ In Vancouver we have broadened the selection criteria to include more patients, so that up to $50 \%$ of symptomatic patients referred are eligible for treatment. Our criteria are as follows:

(a) Minimum stone size of $5 \mathrm{~mm}$, with no maximum size;

(b) Maximum number of six stones;

(c) Minimum of $20 \%$ gall bladder contractility on ultrasound after a fatty meal to assure patency of the cystic duct.

An oral cholecystogram is performed only if there is less than $20 \%$ gall bladder contractility, to assess further gall bladder function. Patients with calcified stones seen on plain abdominal radiography are not excluded.

Unlike other groups we do not use any adjuvant oral chemolitholytic agents, so that clearance of gall stone fragments is by gall bladder expulsion rather than by dissolution.
There have been several long term studies investigating the persistence of abdominal symptoms after elective cholecystectomy. Because of the short follow up period of most groups performing extracorporeal lithotripsy, however, there have been few reports on symptomatic outcome after cholecystolithotripsy. This study aimed to analyse those patients with stone free gall bladders after lithotripsy to determine the stone recurrence rate and the incidence of symptom relief.

\section{Methods}

Two hundred and twenty nine patients were treated with a Lithostar Plus System (Siemens, Erlangen, West Germany), and 47 of them are currently in treatment. There were 167 women and 62 men with an age range of 14-84 years (mean 50 years, median 51 years). Stone numbers ranged from one to six (mean $2 \cdot 0$, median $1 \cdot 0)$, and 147 patients ( $64 \%$ ) had solitary stones. Stone size ranged from 5-44 mm (mean 16.6 $\mathrm{mm}$, median $16 \mathrm{~mm})$. In 40 patients (17\%) calcified stones were seen on plain abdominal radiography. Patients were treated in the supine oblique position by the sonographically guided overhead module of a Lithostar Plus.

Forty six patients, rendered stone free after cholecystolithotripsy, have been followed for a period of two to 25 months (mean 11 months, median 10 months). There were 32 women and 14 men with an age range of 24-80 years (mean 51 years, median 49 years). Thirty eight $(83 \%)$ patients had a solitary calculus, while the remaining eight patients had between two and four stones. Stone size ranged from 5 to $30 \mathrm{~mm}$ (mean $18 \mathrm{~mm}$, median $16 \mathrm{~mm}$ ). Five patients had homogeneously calcified stones seen on plain abdominal radiography. Between one and six treatment sessions were given (mean 2.5, median 2 ). The number of shocks administered ranged from 450 to 22600 (average 7320, median 5200). Intravenous analgesia was used in 40 patients $(87 \%)$, with no analgesic requirements in the remaining six patients, allowing all 46 patients to be treated as outpatients. No patients were given adjuvant oral chemolitholysis under our existing protocol.

All patients had two separate confirmatory ultrasound scans within two weeks when initially diagnosed as fragment free. Patient follow up consisted of monthly ultrasound scans for the first three months, three monthly scans for one year, and six monthly scans for up to two years. Using a standard questionnaire, all 46 patients were interviewed before treatment by the same gastroenterologist to assess the clinical and radiological criteria for eligibility. The same 
questionnaire was used to assess patients' symptoms after gall bladder clearance by a single radiologist $(\mathrm{SHL})$ on the lithotripsy panel.

\section{Results}

Seven of the 46 patients (15\%) whose gall stones were successfully fragmented and cleared developed recurrent calculi. Before treatment all seven patients had solitary calculi, two of which were calcified. Six of the seven patients reformed between two and three calculi and one patient reformed a solitary calculus. All the stones when detected measured less than $10 \mathrm{~mm}$ in maximum diameter. Six patients remain totally asymptomatic, while one describes intermittent right upper quadrant pain no different from that before treatment. This pain had been relieved during a three month stone free period.

Twenty six of the 39 patients $(67 \%)$ whose gall bladders remain stone free at follow up with ultrasound are asymptomatic. Six patients (15\%) complained of some persistent right upper quadrant or epigastric abdominal pain. One patient developed biliary colic because of a bile duct stone, which was diagnosed on endoscopic retrograde cholangiopancreatography (ERCP) and successfully extracted after sphincterotomy. The pain in the other five patients was similar to that before lithotripsy treatment and both interviewers agreed that in these patients the history was compatible with a diagnosis of chronic cholecystitis.

Seven other patients (18\%), although rendered pain free, still complained of various abdominal symptoms including bloating, cramps, indigestion, nausea, and dyspepsia. These atypical biliary symptoms were all present before treatment and may not be related to the biliary tract.

\section{Discussion}

The abdominal symptoms described by patients with symptomatic gall stones may also occur in a variety of other abdominal diseases, in particular peptic ulcer disease, pancreatitis, reflux oesophagitis, irritable bowel syndrome, and diverticular disease. The symptoms of classic biliary colic probably occur in a minority of patients with symptomatic gall stones. Pain from biliary colic may not only be present in the right upper quadrant but anywhere within the abdomen and may radiate to most sites in the back and chest. ${ }^{2}$ It is therefore difficult to select for treatment only those patients with gall stones whose symptoms are attributable to their underlying biliary tract disease.

Most clinicians will recommend treatment for patients with symptomatic gall stones, usually by cholecystectomy, which still remains the gold standard. Several studies after elective cholecystectomy, however, report persistence of abdominal symptoms in up to $50 \%$ of patients..$^{3-11}$ In our group of 46 symptomatic patients treated by cholecystolithotripsy, $70 \%$ have become asymptomatic, including six of seven patients with recurrent calculi. This incidence compares favourably with the outcome after cholecystectomy. One patient with recurrent biliary colic was discovered to have a bile duct stone at ERCP, and we would recommend this procedure in those patients with a high clinical index of suspicion for duct stones. ${ }^{12}$

A major drawback with lithotripsy treatment is that in most series only a minority of patients are eligible for treatment, mainly due to limitations set on stone numbers. In Vancouver, however, by accepting a greater number of stones with no maximum limit on stone size, far more patients are eligible for treatment. Although we do not administer adjuvant oral chemolitholysis, we have reported a $60 \%$ overall fragment clearance at 12 months after successful gall stone fragmentation, which is comparable with results from other centres that do use these agents. ${ }^{13}$ To achieve this result we administer up to 20000 shocks over a maximum of six treatment sessions in order to reduce fragment size to $\leq 3 \mathrm{~mm}$. The number of shocks and treatment sessions are necessarily greater than in other reported series. ${ }^{14-16}$ It is hoped that fragments of such small size will pass from the gall bladder and biliary tract into the duodenum. Some lithotripsy groups using adjuvant oral chemolitholysis end treatment with stone fragments measuring up to $5 \mathrm{~mm}$ in size, thus relying on dissolution to rid the gall bladder of these larger fragments. ${ }^{1+16} \mathrm{It}$ has become clear that because repeated lithotripsy sessions are often necessary, patients must be well motivated to complete their treatment, otherwise the outcome will inevitably be disappointing.

Although the follow up time of the 46 patients reported is short - that is a mean of 11 months - it seems that the patients most suitable for this form of treatment are those with a solitary calculus less than $2 \mathrm{~cm}$ in size. This confirms the findings in other series.' We have also shown, however, that calcified gall stones can also be successfully fragmented and cleared, although one of five patients with these developed an asymptomatic recurrent stone 11 months after completing treatment.

The $15 \%$ incidence of recurrent calculi within one year is similar to that reported by groups using oral bile acid dissolution alone ${ }^{1718}$ and is also similar to the recurrence rate reported after combined lithotripsy treatment with oral bile acids. ${ }^{19}$ We believe that there is little evidence as yet that shows the benefit of adjuvant oral chemolitholysis in clearing the gall bladder of fragments, providing enough shocks are used to fragment stones to $3 \mathrm{~mm}$ or less in size. The data presented here also suggest that the addition of oral dissolution agents may not adversely affect the rate of gall stone recurrence, although further long term follow up is required with greater numbers of patients. In addition, the inhibitory effect of these agents on gall bladder contraction may be counterproductive in expelling stone fragments. ${ }^{20}$ One recent study, however, has shown that impaired gall bladder motility is not improved after gall stone clearance with lithotripsy treatment. ${ }^{21}$ What effect this impaired gall bladder motility will have on gall stone recurrence rates in the long term is yet to be determined. The finding of recurrent calculi should not deter patients or clinicians from undergoing cholecystolithotripsy as only one of seven patients with recurrent gall stones developed 
recurrent symptoms. This finding is in keeping with two other studies which reported that up to $80 \%$ of patients with untreated gall stones may remain asymptomatic over a five to 15 year period. 2223

Patients who develop recurrent symptomatic stones after successful cholecystolithotripsy are eligible for further lithotripsy treatment. The $30 \%$ overall incidence of persistent abdominal symptoms is similar to that after cholecystectomy. These patients require further investigation to exclude other organic diseases. Would they have been better off had they undergone cholecystectomy?

Thanks to Betty Fowler for preparing the manuscript.

1 Sackmann M, Delius M, Sauerbruch T, et al. Shock-wave lithotripsy of gallbladder stones: the first 175 patients. lithotripsy of gallbladder stones:

2 Jorgensen T. Abdominal symptoms and gallstone disease; an epidemiological investigation. Hepatology 1989; 9: 856-60.

3 Bates T, Mercer JC, Harrison M. Symptomatic gallstone disease: before and after cholecystectomy. Gut 1986; 25 : A579-80.

4 Ros E, Zambon D. Postcholecystectomy symptoms: A prospective study of gallstone patients before and two years after surgery. Gut 1987; 28: 1500-4.

5 Rhodes M, Lennard TW. Satisfaction after cholecystectomy. Lancet 1988; i: 599.

6 Bodvall B, Overgaard B. Computer analysis of postcholecystectomy biliary tract syndromes. Surg Gynecol cholecystectomy biliary

7 Gunn A, Keddie N. Some clinical observations on patients with gallstones. Lancet 1972; ii: $239-41$.

8 Bouchier IAD, Rhodes K, Brien MA. A study of symptomatic and 'silent' gallstones. Scand $\mathcal{F}$ Gastroenterol 1968; 3: 299 304.
9 Stefanini P, Carboni M, Patrassi N, et al. Factors influencing the long term results of cholecystectomy. Surg Gynecol Obste 1974; 139: 734-8.

10 Tondelli P, Gyr K, Stalder GA, Allgower M. Post surgica syndromes: the biliary tract. Clin Gastroenterol 1974; 8: 487504.

11 Johnson AG. Cholecystectomy and gallstone dyspepsia. Clinical and physiological study of a symptom complex. Ann R Coll Surg 1975; 56: 69-80

12 Lee SH, Burhenne HJ. Gallbladder surgery following cholecystolithotripsy: suggested guidelines for treatment. $\mathrm{Br} \mathcal{F}$ Systolithotripsy: sugges

13 Fache JS, Rawat B, Burhenne HJ. Biliary extracorporeal lithotripsy without oral chemolitholysis: The Vancouver

Experience in 300 patients. Radiology 1990; 177: 719-21.
14 Ponchon T, Barkun AN, Pujol B, Mestas JL, Lambert R Gallstone disappearance after extracorporeal lithotripsy and Gallstone disappearance after extracorporeal lithotripsy and
oral bile acid dissolution. Gastroenterology 1989; 97: 457-63.

15 Delmont JP, Magnier M, Mosnier H, et al. Results of gallstone lithotripsy in 212 patients using the EDAP LT.01. In: Ferrucci JT, Delius M, Burhenne HJ, eds. Biliary lithotripsy. Chicago: Year Book Medical Publishers Inc, 1989: 95-102.

16 Bory RM. Biliary lithotripsy with the Direx Tripter XI. In: Burhenne HJ, Paumgartner G, Ferrucci JT, eds. Biliary lithotripsy II. Chicago: Year Book Medical Publishers Inc, 1990: 89-92.

17 O'Donnell LDJ, Heaton KW. Recurrence and re-recurrence of gallstones after medical dissolution: a long term followof gallstones after medica

18 Villonova N, Bazzolli F, Toroni F, et al. Gallstone recurrence after successful oral bile acid treatment. A 12-year follow-up study and evaluation of long-term post dissolution treatment. Gastroenterology 1989; 97: 726-31.

19 Sackmann M, Ippisch I, Sauerbruch T, et al. Early gallstone recurrence after successful shock wave therapy. Gastroenterology 1990; 98: 392-6.

20 Forgacs IC, Maisey MN, Murphy GM, Dowling RH Influence of gallstones and ursodeoxycholic acid therapy on gallbladder emptying. Gastroenterology 1984; 87: 299-307.

21 Spengler U, Sackmann M, Sauerbruch T, Holl J, Paumgartner G. Gallbladder motility before and after extracorporeal G. Gallbladder motility before and after extracorporeal

22 Barbara L, Sama C, Moreselli-Labate AM, et al. A population study on the prevalence of gallstone disease: the Sirmione Study. Hepatology 1987; 7: 913-7.

23 Gracie WA, Ranshoff DF. The natural history of silen gallstones. N Engl f Med 1982; 307: 798-800. 